\title{
AN INTEGRAL INEQUALITY WITH APPLICATIONS
}

\author{
$\mathrm{BY}$
}

\author{
M. A. LECKBAND
}

\begin{abstract}
Using a technical integral inequality, J. Moser proved a sharp result on exponential integrability of a certain space of Sobolev functions. In this paper, we show that the integral inequality holds in a general setting using nonincreasing functions and a certain class of convex functions. We then apply the integral inequality to extend the above result by J. Moser to other spaces of Sobolev functions. A second application is given generalizing some different results by $\mathrm{M}$. Jodeit.
\end{abstract}

1. The integral inequality (Theorem 3) presented in this paper has been of interest ever since a simple version of it was proven in 1971 by J. Moser. Subsequent improvements were made by M. Jodeit in 1972, B. F. Jones in 1979 and C. J. Neugebauer in 1980.

Let $1 \leqslant q<\infty, 1 / p+1 / q=1, f \in L^{q}[0, \infty)$ and $\|f\|_{q} \leqslant 1$. Let $\phi \geqslant 0$ be locally integrable, and define

$$
\psi(x)=\left[\int_{0}^{x} \phi^{p}(y) d y\right]^{1 / p} \text { and } F(x)=\int_{0}^{x} f(y) \phi(y) d y .
$$

Let $\Phi$ be a nonincreasing function on [ $0, \infty)$.

In this paper we investigate for which real-valued functions $N(x)$ we get the inequality

$$
\int_{0}^{\infty} \phi\{N[\psi(x)]-N[F(x)]\} d N[\psi(x)] \leqslant C\|\Phi\|_{1} .
$$

Theorem 2 is basic to our proof of this inequality, and a simple calculation involving a concave function shows that the natural functions to consider are convex functions. Moreover, Theorem 1 shows that Theorem 2 is true for general $\phi$ and $f$ if and only if $N(x)$ is a certain type of convex function called a $C^{*}$-convex function.

Finally in $\S \S 5$ and 6 we improve upon the applications given by J. Moser and M. Jodeit, respectively.

2. Definition 1. A continuous function $\rho:[0, \infty) \rightarrow[0, \infty)$ will be called a $C^{*}$-function provided there exists a constant $C_{\rho}<\infty$ such that for $0<d<\infty$, we have a constant $C(d)<\infty$ with

$$
\rho\{(l+d) s\} \leqslant C_{\rho} \cdot\{(l-1) s\},
$$

for all $l>C(d)$ and $0<s<\infty$.

Received by the editors March 26, 1982 and, in revised form, March 21, 1983.

1980 Mathematics Subject Classification. Primary 42A96. 
We note that if $\rho:[0, \infty) \rightarrow[0, \infty)$ is a concave nondecreasing function, then $\rho$ is a $C^{*}$-function. Moreover, the set of $C^{*}$-functions is closed under addition and multiplication; hence, all polynomials with positive coefficients are $C^{*}$-functions.

Definition 2. A function $N:[0, \infty) \rightarrow[0, \infty)$ will be called a $C^{*}$-convex function if $N(0)=0, N$ is convex, $N \in C^{\mathrm{l}}[0, \infty)$, and $\rho$ defined by the differential equation $\rho(N(x))=N^{\prime}(x)$ is a $C^{*}$-function.

Some common examples of $C^{*}$-convex functions are $N(x)=e^{x}-1, N(x)=e^{x^{2}}$ -1 , and $N(x)=x^{p}, p \geqslant 1$.

The following theorem gives an equivalent condition that a convex function must satisfy to be $C^{*}$-convex.

THEOREM 1. Let $N \in C^{1}[0, \infty)$ be a nonnegative convex function and $N(0)=0$. Then $N$ is a $C^{*}$-convex function if and only if there are constants $0<c, d<\infty$, such that for $l>d, 0<s<\infty$, we have

$$
\left[N^{-1}(l s)\right]^{2} \leqslant\left[N^{-1}\{(l-1) s\}\right]\left[N^{-1}\{(l+c) s\}\right] .
$$

Proof. First assume that $N$ is a $C^{*}$-convex function; that is, $N(0)=0, N \in$ $C^{1}[0, \infty), N \geqslant 0$, and the function $\phi$ defined by $\phi(N(x))=N^{\prime}(x)$ is a $C^{*}$-function.

If $(*)$ does not hold, then for any $c>0$ there is sequence $\left\{l_{n} s_{n}\right\}$ such that:

(i) $l_{n} \rightarrow \infty$,

(ii) $\left[N^{-1}\left(l_{n} s_{n}\right)\right]^{2}>\left[N^{-1}\left(\left\{l_{n}-1\right\} s_{n}\right)\right]\left[N^{-1}\left(\left\{l_{n}+c\right\} s_{n}\right)\right]$.

We will show that for some $a, c / 2<a<c$, we get

$$
\phi\left(\left\{l_{n}+a\right\} s_{n}\right) \geqslant \frac{c}{4} \phi\left(\left\{l_{n}-1\right\} s_{n}\right) .
$$

This will imply that $\phi$ is not a $C^{*}$-function, hence contradicting the assumption that $N$ is $C^{*}$-convex.

We calculate from $N^{\prime}(x)=\phi(N(x))$ that $\left(N^{-1}\right)^{\prime}(y)=1 / \phi(y)$; therefore,

$$
N^{-1}\left(\left\{l_{n}+c\right\} s_{n}\right)=\int_{l_{n} s_{n}}^{\left(l_{n}+c\right) s_{n}} \frac{1}{\phi(y)} d y+N^{-1}\left(l_{n} s_{n}\right)
$$

and

$$
N^{-1}\left(\left\{l_{n}-1\right\} s_{n}\right)=N^{-1}\left(l_{n} s_{n}\right)-\int_{\left(l_{n}-1\right) s_{n}}^{l_{n} s_{n}} \frac{1}{\phi(y)} d y .
$$

We use the above two expressions to reduce inequality (ii) to

$$
N^{-1}\left(l_{n} s_{n}\right) \int_{\left(l_{n}-1\right) s_{n}}^{l_{n} s_{n}} \frac{1}{\phi(y)} d y>N^{-1}\left\{\left(l_{n}-1\right) s_{n}\right\} \cdot \int_{l_{n} s_{n}}^{\left(l_{n}+c\right) s_{n}} \frac{1}{\phi(y)} d y .
$$

By the way $\phi$ is defined, $1 / \phi(y)$ is nonincreasing and we see

$$
N^{-1}\left(l_{n} s_{n}\right) \cdot \frac{s_{n}}{\phi\left\{\left(l_{n}-1\right) s_{n}\right\}} \geqslant N^{-1}\left(l_{n} s_{n}\right) \int_{\left(l_{n}-1\right) s_{n}}^{l_{n} s_{n}} \frac{1}{\phi(y)} d y,
$$

and for any $c / 2<a<c$,

$$
N^{-1}\left\{\left(l_{n}-1\right) s_{n}\right\} \int_{l_{n} s_{n}}^{\left(l_{n}+c\right) s_{n}} \frac{1}{\phi(y)} d y \geqslant N^{-1}\left\{\left(l_{n}-1\right) s_{n}\right\} \cdot \frac{s_{n}}{\phi\left\{\left(l_{n}+a\right) s_{n}\right\}} \cdot \frac{c}{2} .
$$


Hence (A), (B) and (C) imply

$$
\phi\left\{\left(l_{n}+a\right) s_{n}\right\} \geqslant \frac{N^{-1}\left\{\left(l_{n}-1\right) s_{n}\right\}}{N^{-1}\left(l_{n} s_{n}\right)} \frac{c}{2} \phi\left\{\left(l_{n}-1\right) s_{n}\right\} .
$$

The mean value theorem implies, since $N^{-1}(0)=0$,

$$
\frac{N^{-1}\left\{\left(l_{n}-1\right) s_{n}\right\}}{N^{-1}\left(l_{n} s_{n}\right)}=\frac{\left(N^{-1}\right)^{\prime}(\xi)}{\left(N^{-1}\right)^{\prime}\left(\xi_{+}\right)} \frac{\left(l_{n}-1\right) s_{n}}{l_{n} s_{n}}, \quad \text { where } 0<\xi \leqslant \xi_{+} .
$$

$N^{-1}$ is concave, so $\left(N^{-1}\right)^{\prime}(\xi) \geqslant\left(N^{-1}\right)^{\prime}\left(\xi_{+}\right)$and inequality (D) becomes

$$
\phi\left\{\left(l_{n}+a\right) s_{n}\right\} \geqslant \frac{\left(l_{n}-1\right)}{l_{n}} \frac{c}{2} \phi\left\{\left(l_{n}-1\right) s_{n}\right\},
$$

and for $l_{n}$ large, $\left(l_{n}-1\right) / l_{n} \geqslant 1 / 2$. Thus $\phi$ is not a $C^{*}$-function.

We assume now that $(*)$ holds and show that $\phi$ is a $C^{*}$-function by contradiction.

Suppose $\left\{l_{n} s_{n}\right\}$ is a sequence for which the following is true.

Given $0<c, d<\infty$, then:

(a) $l_{n} \rightarrow \infty$.

(b) $\phi\left\{\left(l_{n}+d\right) s_{n}\right\}>c \phi\left\{\left(l_{n}-1\right) s_{n}\right\}$.

We will show that there exists a sequence $\left\{\bar{l}_{n} \bar{s}_{n}\right\}$ with

$$
\left[N^{-1}\left(\bar{l}_{n} \bar{s}_{n}\right)\right]^{2}>\left[N^{-1}\left\{\left(\bar{l}_{n}-1\right) \bar{s}_{n}\right\}\right]\left[N^{-1}\left\{\left(\bar{l}_{n}+a\right) \bar{s}_{n}\right\}\right],
$$

where $a=c / 2$. This contradicts $(*)$.

From above, inequality (E) will be true if

$$
\int_{\left(\bar{l}_{n}-1\right) \bar{s}_{n}}^{\bar{l}_{n} \bar{s}_{n}} \frac{1}{\phi(x)} d x \geqslant \int_{\bar{l}_{n} \bar{s}_{n}}^{\left(\bar{l}_{n}+a\right) \bar{s}_{n}} \frac{1}{\phi(x)} d x .
$$

To achieve (**), for suitable $\alpha$ and $d$ let

$$
\bar{l}_{n} \bar{s}_{n}=\left(l_{n}+d\right) s_{n} \text { and }\left(\bar{l}_{n}-1\right) \bar{s}_{n}=\left(l_{n}-1\right) s_{n}-\alpha s_{n} .
$$

Then

$$
\bar{s}_{n}=(d+1+\alpha) s_{n} \text { and } \bar{l}_{n}=\left(l_{n}+d\right) /(d+1+\alpha) .
$$

To determine suitable $\alpha$ and $d$, we observe

$$
\frac{a \bar{s}_{n}}{\phi\left\{\left(l_{n}+d\right) s_{n}\right\}} \geqslant \int_{\bar{l}_{n} \bar{s}_{n}=\left(l_{n}+d\right) s_{n}}^{\left(\bar{l}_{n}+a\right) \bar{s}_{n}} \frac{1}{\phi(x)} d x
$$

and

$$
\int_{\left(\bar{l}_{n}-1\right) \bar{s}_{n}}^{\bar{l}_{n} \bar{s}_{n}} \frac{1}{\phi(x)} d x \geqslant \int_{\left(l_{n}-1-\alpha\right) s_{n}}^{\left(l_{n}-1\right) s_{n}} \frac{1}{\phi(x)} d x \geqslant \frac{\alpha s_{n}}{\phi\left\{\left(l_{n}-1\right) s_{n}\right\}} .
$$

The inequality $(* *)$ will be true if we have

$$
\frac{\alpha s_{n}}{\phi\left\{\left(l_{n}-1\right) s_{n}\right\}} \geqslant a(d+1+\alpha) \frac{s_{n}}{\phi\left\{\left(l_{n}+d\right) s_{n}\right\}}
$$

or

$$
\phi\left\{\left(l_{n}+d\right) s_{n}\right\} \geqslant \frac{a(d+1+\alpha)}{\alpha} \phi\left\{\left(l_{n}-1\right) s_{n}\right\} .
$$


From hypothesis, this is true if we require that $a=c / 2, \alpha=a+1$, and $d$ be some fixed constant greater than $c / 2$.

3. Let $(x, \mathfrak{T}, \mu)$ be a measure space and, for $0<r<\infty$, let $S_{r} \in$ Or such that $S_{r^{\prime}} \subset S_{r^{\prime \prime}}$ if $r^{\prime} \leqslant r^{\prime \prime}$ and $S_{0}$ is the empty set. For $\phi \geqslant 0, f \geqslant 0,1 \leqslant q<\infty$ and $1 / q+1 / p=1$ we will write $\phi_{r}=\phi \cdot \chi_{S_{r}}, \psi(r)=\left\|\phi_{r}\right\|_{p, \mu}$ and $F(r)=\int_{X} f \cdot \phi_{r} d \mu$. In addition, we always assume $\|f\|_{q, \mu} \leqslant 1$ and $\psi(r)<\infty$ for $0<r<\infty$.

Lemma 1. Let $N \in C^{1}[0, \infty), N \geqslant 0, N$ convex, and $N(0)=0$. If $1 \leqslant q<\infty$, then there is a constant $C, 2<C<\infty$, which depends only upon $q$, such that

$$
N\left\{\psi\left(r_{1}\right)\right\} \geqslant C \max _{j=1.2}\left[N\left\{\psi\left(r_{j}\right)\right\}-N\left\{F\left(r_{j}\right)\right\}\right]
$$

implies $\psi\left(r_{2}\right) \leqslant 2 \psi\left(r_{1}\right)$.

Proof. This proof is done by assuming $1<q<\infty$ and a similar argument proves $1=q$. Let $1<q<\infty, s=\max _{j=1,2}\left[N\left\{\psi\left(r_{j}\right)\right\}-N\left\{F\left(r_{j}\right)\right\}\right]$ and assume $r_{2} \geqslant r_{1}$.

(i) $\left\|\phi_{r_{2}}-\phi_{r_{1}}\right\|_{p} \leqslant p^{1 / p} \psi^{1 / q}\left(r_{2}\right)\left\{\psi\left(r_{2}\right)-\psi\left(r_{1}\right)\right\}^{1 / p}$.

To demonstrate (i) we use the definition of $\phi_{r}$ to get

$$
\begin{aligned}
\left\|\phi_{r_{2}}-\phi_{r_{1}}\right\|_{p}^{p} & =\int \phi_{r_{2}}^{p}-\phi_{r_{1}}^{p} d \mu=\psi^{p}\left(r_{2}\right)-\psi^{p}\left(r_{1}\right) \\
& \leqslant p \psi^{p-1}\left(r_{2}\right)\left[\psi\left(r_{2}\right)-\psi\left(r_{1}\right)\right] .
\end{aligned}
$$

The last inequality uses the mean value theorem.

(ii) $\left\|f \cdot \chi_{X \backslash S_{1}}\right\|_{q} \leqslant\left(q s / N\left\{\psi\left(r_{1}\right)\right\}\right)^{1 / q}$, if $N\left\{\psi\left(r_{1}\right)\right\}>2 s$, where $S_{1}=S_{r_{1}}$.

To prove (ii), Hölder's inequality, $\left\|f \cdot \chi_{S_{1}}\right\|_{q} \leqslant 1$, and the convexity of $N$, imply

$$
N\left\{F\left(r_{1}\right)\right\} \leqslant N\left\{\left\|f \cdot \chi_{S_{1}}\right\|_{q} \psi\left(r_{1}\right)\right\} \leqslant\left\|f \cdot \chi_{S_{1}}\right\|_{q} N\left\{\psi\left(r_{1}\right)\right\},
$$

so

$$
N\left\{\psi\left(r_{1}\right)\right\} \leqslant s+N\left\{F\left(r_{1}\right)\right\} \leqslant s+\left\|f \cdot \chi_{s_{1}}\right\|_{q} N\left\{\psi\left(r_{1}\right)\right\} .
$$

Rearranging the terms we get

$$
\left(1-\frac{s}{N\left\{\psi\left(r_{1}\right)\right\}}\right)^{q} \leqslant\left\|f \cdot \chi_{S_{1}}\right\|_{q}^{q} \leqslant 1-\left\|f \cdot \chi_{X \backslash S_{1}}\right\|_{q}^{q} .
$$

Now we apply the mean value theorem to $x^{q}$, for $0<x<1$,

$$
\left\|f \cdot \chi_{X \backslash s_{1}}\right\|_{q}^{q} \leqslant 1-\left(1-\frac{s}{N\left\{\psi\left(r_{1}\right)\right\}}\right)^{q} \leqslant \frac{q s}{N\left\{\psi\left(r_{1}\right)\right\}} .
$$

This proves (ii).

To prove the lemma assume $N\left\{F\left(r_{2}\right)\right\} \geqslant N\left\{\psi\left(r_{1}\right)\right\}$; if not, we calculate

$$
N\left\{\psi\left(r_{1}\right)\right\} \geqslant C N\left\{\psi\left(r_{2}\right)\right\}-C N\left\{F\left(r_{2}\right)\right\}
$$

or

$$
N\left\{\frac{(C+1)}{C} \psi\left(r_{1}\right)\right\} \geqslant\left(\frac{C+1}{C}\right) N\left\{\psi\left(r_{1}\right)\right\} \geqslant N\left\{\psi\left(r_{2}\right)\right\} .
$$

Hence $2 \psi\left(r_{1}\right) \geqslant(C+1) \psi\left(r_{1}\right) / C \geqslant \psi\left(r_{2}\right)$, and the lemma is proved. 
We start with

$$
N\left\{\psi\left(r_{2}\right)\right\}-N\left\{\psi\left(r_{1}\right)\right\} \leqslant s+N\left\{F\left(r_{2}\right)\right\}-N\left\{F\left(r_{1}\right)\right\} .
$$

The mean value theorem and the convexity of $N$ imply

$$
\begin{gathered}
N\left\{\psi\left(r_{2}\right)\right\}-N\left\{\psi\left(r_{1}\right)\right\}=N^{\prime}(\eta)\left\{\psi\left(r_{2}\right)-\psi\left(r_{1}\right)\right\}, \\
N\left\{F\left(r_{2}\right)\right\}-N\left\{F\left(r_{1}\right)\right\}=N^{\prime}(\xi)\left\{F\left(r_{2}\right)-F\left(r_{2}\right)\right\}, \\
N^{\prime}(\eta) \geqslant N^{\prime}(\xi), \quad \eta \geqslant \xi \text { and } \eta \geqslant \psi\left(r_{1}\right) .
\end{gathered}
$$

Inequality (A) now becomes

$$
N^{\prime}(\eta)\left(\psi\left(r_{2}\right)-\psi\left(r_{1}\right)\right) \leqslant s+N^{\prime}(\eta)\left[F\left(r_{2}\right)-F\left(r_{1}\right)\right] .
$$

Hölder's inequality (i), and (ii) are applied to $F\left(r_{2}\right)-F\left(r_{1}\right)$ to get

$$
\begin{aligned}
F\left(r_{2}\right)-F\left(r_{1}\right) & \leqslant\left\|f \cdot \chi_{X \backslash S_{1}}\right\|_{q}\left\|\phi_{r_{2}}-\phi_{r_{1}}\right\|_{p} \\
& \leqslant p^{1 / p}\left(\frac{q s}{N\left\{\psi\left(r_{1}\right)\right\}}\right)^{1 / q}\left(\psi\left(r_{2}\right)\right)^{1 / q} \cdot\left(\psi\left(r_{2}\right)-\psi\left(r_{1}\right)\right)^{1 / p} .
\end{aligned}
$$

We now apply Young's inequality on the right-hand side:

$$
F\left(r_{2}\right)-F\left(r_{1}\right) \leqslant \frac{p^{q / p} s \psi\left(r_{2}\right)}{N\left\{\psi\left(r_{1}\right)\right\}}+\frac{\left(\psi\left(r_{2}\right)-\psi\left(r_{1}\right)\right)}{p} .
$$

Inequality (B) now becomes

$$
N^{\prime}(\eta)\left(\psi\left(r_{2}\right)-\psi\left(r_{1}\right)\right) \leqslant s+\frac{N^{\prime}(\eta) \psi\left(r_{2}\right) s}{N\left\{\psi\left(r_{1}\right)\right\}} \cdot p^{q / p}+\frac{N^{\prime}(\eta)\left(\psi\left(r_{2}\right)-\psi\left(r_{1}\right)\right)}{p} .
$$

Rearranging terms we get

$$
\psi\left(r_{2}\right)\left(1-\frac{q p^{q / p_{S}}}{N\left\{\psi\left(r_{1}\right)\right\}}\right) \leqslant\left(1+\frac{q s}{\psi\left(r_{1}\right) N^{\prime}(\eta)}\right) \cdot \psi\left(r_{1}\right) .
$$

Since $\eta \geqslant \psi\left(r_{1}\right)$ implies $\psi\left(r_{1}\right) N^{\prime}(\eta) \geqslant N\left\{\psi\left(r_{1}\right)\right\}$, the choice $N \psi\left\{\left(r_{1}\right)\right\} \geqslant$ $\left(q+2 q p^{q / p}\right) s$, i.e., $C=q+2 q p^{q / p}$, will complete the proof.

THEOREM 2. Let $N$ be a $C^{*}$-convex function. There is a constant $C, 2<C<\infty$, which depends only upon $N$ and $q, 1 \leqslant q<\infty$, such that

$$
N\left\{\psi\left(r_{1}\right)\right\} \geqslant C \max _{j=1,2} N\left\{\psi\left(r_{j}\right)\right\}-N\left\{F\left(r_{j}\right)\right\}
$$

implies

$$
N\left\{\psi\left(r_{2}\right)\right\}-N\left\{\psi\left(r_{1}\right)\right\} \leqslant C \max _{j=1,2} N\left\{\psi\left(r_{j}\right)\right\}-N\left\{F\left(r_{j}\right)\right\} .
$$

Proof. This is done by assuming $1<q<\infty$ and a similar proof proves $q=1$. then

Assume $1<q<\infty, r_{2} \geqslant r_{1}$ and $N\left\{F\left(r_{2}\right)\right\} \geqslant N\left\{\psi\left(r_{1}\right)\right\}$. If $N\left\{F\left(r_{2}\right)\right\} \leqslant N\left\{\psi\left(r_{1}\right)\right\}$,

$$
N\left\{\psi\left(r_{2}\right)\right\}-N\left\{\psi\left(r_{1}\right)\right\} \leqslant N\left\{\psi\left(r_{2}\right)\right\}-N\left\{F\left(r_{2}\right)\right\}
$$

and we are done. 
Let $s=\max _{j=1,2}\left\{N\left\{\psi\left(r_{j}\right)\right\}-N\left\{F\left(r_{j}\right)\right\}\right\}$. The following inequalities are used:

(i) $\left\|\phi_{r_{2}}-\phi_{r_{1}}\right\|_{p} \leqslant p^{1 / p} \psi^{1 / q}\left(r_{2}\right)\left[\psi\left(r_{2}\right)-\psi\left(r_{1}\right)\right]^{1 / p}$.

(ii) $\left\|f \cdot \chi_{X \backslash S_{1}}\right\|_{q} \leqslant q^{1 / q}\left[1-N^{-1}\left\{N\left[\psi\left(r_{1}\right)\right]-s\right\} / \psi\left(r_{1}\right)\right]^{1 / q}$.

The first inequality has already been shown, and to prove (ii) we start with

$$
N\left\{\psi\left(r_{1}\right)\right\} \leqslant s+N\left\{F\left(r_{1}\right)\right\} \leqslant s+N\left\{\left\|f \cdot \chi_{s_{1}}\right\|_{q} \psi\left(r_{1}\right)\right\},
$$

by Hölder's inequality. Thus

$$
N^{-1}\left[N\left\{\psi\left(r_{1}\right)\right\}-s\right] \leqslant \psi\left(r_{1}\right) \cdot\left\|f \cdot \chi_{s_{1}}\right\|_{q} .
$$

Since $\left\|f \cdot \chi_{S_{1}}\right\|_{q}^{q} \leqslant 1-\left\|f \cdot \chi_{X \backslash S_{1}}\right\|_{q}^{q}$, we derive

$$
\left[\frac{N^{-1}\left\{N\left[\psi\left(r_{1}\right)\right]-s\right\}}{\psi\left(r_{1}\right)}\right]^{q} \leqslant\left\|f \cdot \chi_{s_{1}}\right\|_{q}^{q} \leqslant 1-\left\|f \cdot \chi_{X \backslash S_{1}}\right\|_{q}^{q}
$$

or

$$
\left\|f \cdot \chi_{X \backslash S_{1}}\right\|_{q}^{q} \leqslant 1-\left[\frac{N^{-1}\left\{N\left[\psi\left(r_{1}\right)\right]-s\right\}}{\psi\left(r_{1}\right)}\right]^{q} .
$$

We now apply the mean value theorem to $x^{q}, 0<x<1$, to derive

$$
\left\|f \cdot \chi_{X \backslash S_{1}}\right\|_{q}^{q} \leqslant q\left[1-\frac{N^{-1}\left\{N\left[\psi\left(r_{1}\right)\right]-s\right\}}{\psi\left(r_{1}\right)}\right] .
$$

This is (ii).

The mean value theorem and the convexity of $N$ imply

(iii)

$$
\begin{aligned}
& N^{\prime}(\eta)\left[\psi\left(r_{2}\right)-\psi\left(r_{1}\right)\right]=N\left\{\psi\left(r_{2}\right)\right\}-N\left\{\psi\left(r_{1}\right)\right\}, \\
& N^{\prime}(\zeta)\left[F\left(r_{2}\right)-F\left(r_{1}\right)\right]=N\left\{F\left(r_{2}\right)\right\}-N\left\{F\left(r_{1}\right)\right\}, \\
& N^{\prime}(\eta) \geqslant N^{\prime}(\zeta) \text { and } n \geqslant \zeta .
\end{aligned}
$$

To prove the theorem we start with

$$
\begin{aligned}
N^{\prime}(\eta)\left[\psi\left(r_{2}\right)-\psi\left(r_{1}\right)\right] & =N\left\{\psi\left(r_{2}\right)\right\}-N\left\{\psi\left(r_{1}\right)\right\} \leqslant s+N\left\{F\left(r_{2}\right)\right\}-N\left\{\psi\left(r_{1}\right)\right\} \\
& \leqslant s+N^{\prime}(\zeta)\left[F\left(r_{2}\right)-F\left(r_{1}\right)\right]
\end{aligned}
$$

and from (iii)

$$
\leqslant s+N^{\prime}(\eta)\left[F\left(r_{2}\right)-F\left(r_{1}\right)\right] .
$$

We recall the definition of $F(r)$ and use Hölder's inequality to derive

$$
F\left(r_{2}\right)-F\left(r_{1}\right) \leqslant\left\|f \cdot \chi_{X \backslash S_{1}}\right\|_{q} \cdot\left\|\phi_{r_{2}}-\phi_{r_{1}}\right\|_{p} .
$$

Thus

$$
N^{\prime}(\eta)\left[\psi\left(r_{2}\right)-\psi\left(r_{1}\right)\right] \leqslant s+N^{\prime}(\eta)\left(\left\|f \cdot \chi_{X \backslash S_{1}}\right\|_{q}\left\|\phi_{r_{2}}-\phi_{r_{1}}\right\|_{p}\right) .
$$

with the right-hand sides of inequalities (i) and (ii) we obtain from $\left(\mathrm{A}^{\prime}\right)$

$$
\begin{aligned}
N^{\prime}(\eta)\left[\psi\left(r_{2}\right)-\psi\left(r_{1}\right)\right] \leqslant & s+p^{1 / p} q^{1 / q} N^{\prime}(\eta)\left[1-\frac{N^{-1}\left\{N\left[\psi\left(r_{1}\right)\right]-s\right\}}{\psi\left(r_{1}\right)}\right]^{1 / q} \\
& \times \psi\left(r_{2}\right)^{1 / q}\left[\psi\left(r_{2}\right)-\psi\left(r_{1}\right)\right]^{1 / q} .
\end{aligned}
$$


We apply Young's inequality to get

$$
\begin{aligned}
N^{\prime}(\eta)\left[\psi\left(r_{2}\right)-\psi\left(r_{1}\right)\right] \leqslant & s p^{q / p} N^{\prime}(\eta)\left[\frac{\psi\left(r_{2}\right)}{\psi\left(r_{1}\right)}\right]\left[\psi\left(r_{1}\right)-N^{-1}\left\{N\left[\psi\left(r_{1}\right)\right]-s\right\}\right] \\
& +\frac{N^{\prime}(\eta)}{p}\left[\psi\left(r_{2}\right)-\psi\left(r_{1}\right)\right] .
\end{aligned}
$$

We require the constant $C$ in the conclusion to be greater than the constant $\bar{C}$ in Lemma 1 . Then $\psi\left(r_{2}\right) / \psi\left(r_{1}\right) \leqslant 2$ and we now have

(*) $\quad N^{\prime}(\eta)\left[\psi\left(r_{2}\right)-\psi\left(r_{1}\right)\right] \leqslant q s+2 q p^{q / p} N^{\prime}(\eta)\left[\psi\left(r_{1}\right)-N^{-1}\left\{N\left[\psi\left(r_{1}\right)\right]-s\right\}\right]$.

Let $\rho(N(x))=N^{\prime}(x)$. Since $N$ is a $C^{*}$-convex function there is a constant $C_{\rho}<\infty$ such that $\rho\{(l+d) s\} \leqslant C_{\rho} \rho\{(l-1) s\}$ for $0<d<\infty, C(d)<l$ and $0<s<\infty$.

Suppose for fixed $a>4 q p^{q / p} C_{\rho}$ we have $N\left\{\psi\left(r_{2}\right)\right\}-N\left\{\psi\left(r_{1}\right)\right\}>$ as. Then $\psi\left(r_{2}\right)$ $\geqslant N^{-1}\left[N\left\{\psi\left(r_{1}\right)\right\}+\right.$ as $]$ and

$$
N^{\prime}(\eta)=\frac{N\left\{\psi\left(r_{2}\right)\right\}-N\left\{\psi\left(r_{1}\right)\right\}}{\psi\left(r_{2}\right)-\psi\left(r_{1}\right)} \leqslant \frac{N\left\{\psi\left(r_{2}\right)\right\}-N\left\{\psi\left(r_{1}\right)\right\}}{N^{-1}\left[N\left\{\psi\left(r_{1}\right)\right\}+a s\right]-\psi\left(r_{1}\right)}
$$

We substitute this expression into the right-hand side of $(*)$ and get

$$
\begin{aligned}
N^{\prime}(\eta)\left[\psi\left(r_{2}\right)-\psi\left(r_{1}\right)\right] \leqslant & q s+2 q p^{q / p}\left[N\left\{\psi\left(r_{2}\right)\right\}-N\left\{\psi\left(r_{1}\right)\right\}\right] \\
& \times\left[\frac{\psi\left(r_{1}\right)-N^{-1}\left\{N\left[\psi\left(r_{1}\right)\right]-s\right\}}{N^{-1}\left[N\left\{\psi\left(r_{1}\right)\right\}+a s\right]-\psi\left(r_{1}\right)}\right] .
\end{aligned}
$$

To the quotient in brackets, we apply the mean value theorem to both numerator and denominator:

$$
\begin{aligned}
\frac{\psi\left(r_{1}\right)-N^{-1}\left\{N\left[\psi\left(r_{1}\right)\right]-s\right\}}{N^{-1}\left\{N\left[\psi\left(r_{1}\right)\right]+a s\right\}-\psi\left(r_{1}\right)} & =\frac{N^{-1}\left\{N\left[\psi\left(r_{1}\right)\right]\right\}-N^{-1}\left\{N\left[\psi\left(r_{1}\right)\right]-s\right\}}{N^{-1}\left\{N\left[\psi\left(r_{1}\right)\right]+a s\right\}-N^{-1}\left\{N\left[\psi\left(r_{1}\right)\right]\right\}} \\
& =\frac{\left(N^{-1}\right)^{\prime}(\delta)}{\left(N^{-1}\right)^{\prime}(\bar{\delta})} \frac{s}{a s}
\end{aligned}
$$

where $N\left[\psi\left(r_{1}\right)\right]-s \leqslant \delta \leqslant N\left[\psi\left(r_{1}\right)\right]$ and $N\left[\psi\left(r_{1}\right)\right] \leqslant \bar{\delta} \leqslant N\left[\psi\left(r_{1}\right)\right]+$ as. Since $\left(N^{-1}\right)^{\prime}$ is a nonincreasing function the right-hand side is

$$
\leqslant \frac{\left(N^{-1}\right)^{\prime}\left(N\left\{\psi\left(r_{1}\right)\right\}-s\right\}}{\left(N^{-1}\right)^{\prime}\left(N\left\{\psi\left(r_{1}\right)\right\}+a s\right)} \cdot \frac{1}{a} .
$$

From $N^{\prime}(x)=\rho(N(x))$ we calculate $\left(N^{-1}\right)^{\prime}(y)=1 / \rho(y)$, and

$$
\frac{\left(N^{-1}\right)^{\prime}\left(N\left\{\psi\left(r_{1}\right)\right\}-s\right)}{\left(N^{-1}\right)^{\prime}\left(N\left\{\psi\left(r_{1}\right)\right\}+a s\right)} \cdot \frac{1}{a}=\frac{\rho\left[N\left\{\psi\left(r_{1}\right)\right\}+a s\right]}{\rho\left[N\left\{\psi\left(r_{1}\right)\right\}-s\right]} \cdot \frac{1}{a} .
$$


We require $N\left\{\psi\left(r_{1}\right)\right\} \geqslant C(a) s$, i.e. the constant $C \geqslant C(a)$, and then since $\rho$ is a $C^{*}$-function the above is less than $C_{\rho} / a$. Thus $(* *)$ becomes

$$
\begin{aligned}
N\left\{\psi\left(r_{2}\right)\right\}-N\left\{\psi\left(r_{1}\right)\right\} & =N^{\prime}(\eta)\left[\psi\left(r_{2}\right)-\psi\left(r_{1}\right)\right] \\
& \leqslant q s+2 q p^{q / p} \frac{C_{\rho}}{a}\left[N\left\{\psi\left(r_{2}\right)\right\}-N\left\{\psi\left(r_{1}\right)\right\}\right] \\
& =q s+\frac{1}{2}\left[N\left\{\psi\left(r_{2}\right)\right\}-N\left\{\psi\left(r_{1}\right)\right\}\right]
\end{aligned}
$$

and the choice of $C>\max \{\bar{C}, C(a)\}$ completes the proof.

It is not possible to improve upon Theorem 2 to include all convex functions. For some simple cases the conclusion of Theorem 2 is equivalent to the inequality used in Theorem 1.

4. TheOREM 3. Let $\Phi:[0, \infty) \rightarrow[0, \infty)$ be nonincreasing, $\psi(r)$ continuous and $N$ a $C^{*}$-convex function. Then there is a constant $C$, which depends only on $N$ and $q$, such that

$$
\int_{0}^{\infty} \Phi\{N[\psi(r)]-N[F(r)]\} d m^{*} \leqslant C \int_{0}^{\infty} \Phi(t) d t,
$$

where $m^{*}$ is the measure induced by $N\{\psi(r)\}$.

Proof. Let

$$
E_{s}=\{r: \Phi\{N[\psi(r)]-N[F(r)]\}>s\}, \quad \Omega(t)=\sup \{u: \Phi(u) \geqslant t\} .
$$

We note that $\Omega(t)$ is the inverse of $\Phi$ and obtain

$$
E_{s} \subset\{r: N[\psi(r)]-N[F(r)] \leqslant \Omega(s)\} .
$$

For $r_{1}, r_{2} \in E_{s}$ and $r_{2} \geqslant r_{1}$ we have

(i) $\max _{j=1.2} N\left[\psi\left(r_{j}\right)\right]-N\left[F\left(r_{j}\right)\right] \leqslant \Omega(s)$.

Theorem 2 says there is a constant $C$, which depends only on $N$ and $q$, such that $N\left\{\psi\left(r_{1}\right)\right\} \geqslant C \Omega(s)$. This and inequality (i) imply

$$
N\left\{\psi\left(r_{2}\right)\right\}-N\left\{\psi\left(r_{1}\right)\right\} \leqslant C \Omega(s) .
$$

This and the continuity of $N[\psi(r)]$ give $m^{*}\left(E_{s}\right) \leqslant 2 C \Omega(s)$. So

$$
\begin{aligned}
\int_{0}^{\infty} \Phi\{N[\psi(r)]-N[F(r)]\} d m^{*} & =\int_{0}^{\infty} m^{*}\left(E_{s}\right) d s, \\
& \leqslant \int_{0}^{\infty} 2 C \Omega(s) d s=2 C \int_{0}^{\infty} \Phi(t) d t,
\end{aligned}
$$

and the theorem is proven.

5. Let $D$ be an open domain in $\mathbf{R}^{n}, n \geqslant 2$, with finite measure. For $1 \leqslant q \leqslant n$, we define $L_{i}^{q}(D)$ to be the space of functions in $L^{q}$ with compact support in $D$, and whose derivatives exist in the weak sense and are functions in $L^{q}$.

Given a measurable function $u: D \rightarrow \mathbf{R}$, let $\lambda_{u}(y)=|\{x \in D:|u(x)|>y\}|$ and $u^{*}(t)=\inf \left\{s: \lambda_{u}(s) \leqslant t\right\}$. The function $u^{*}(t)$ is called the nonincreasing rearrangement of $u$ and a general reference is [1]. 
In the calculations that follow we desire to extend the domain of the rearrangement to $\mathbf{R}^{n}$. Let

$$
u^{\#}(x)=u^{*}\left(\frac{|x|^{n} \omega_{n-1}}{n}\right) \quad \text { for } x \in \mathbf{R}^{n}
$$

Here $\omega_{n-1}$ denotes the area of the unit sphere in $\mathbf{R}^{n}$, and if we denote $D^{\#}$ to be the ball in $\mathbf{R}^{n}$ centered at the origin of measure equal to $D$, then the support of $u^{\#}$ is contained in $D^{\#}$.

We have the following

Lemma A. Let $u \in L^{p}(D), 1 \leqslant p<\infty$. Then

$$
\int_{D^{\#}}\left[u^{\#}(x)\right]^{p} d x=\int_{D}|u(x)|^{p} d x .
$$

LEMMA B. Let $\left\{u_{n}\right\}$ be a sequence of functions in $L^{p}\left(\mathbf{R}^{n}\right), 1 \leqslant p<\infty$, converging to $u$ in $L^{p}$. Then there exists a subsequence $\left\{u_{n_{k}}\right\}$ such that $u_{n_{k}}^{\#}$ converges to $u^{\#}$ a.e.

Lemma B allows us to prove Theorem 4 using sufficiently nice enough functions for which the techniques in G. D. Mostow [5] will prove the following

Lemma C. Let $u \in C_{0}^{\infty}(D)$. Then

$$
\int_{D^{\#}}\left|\nabla\left(u^{\#}(x)\right)\right|^{p} d x \leqslant \int_{D}|\nabla u(x)|^{p} d x \quad \text { for } 1 \leqslant p<\infty .
$$

The following theorem is a generalization of J. Moser [4].

THEOREM 4. Let $N$ be a $C^{*}$-convex function. If $u \in L_{1}^{q}(D), 1 \leqslant q \leqslant n$, such that $\int_{D}|\nabla u(x)|^{q} d x \leqslant 1$, then there exists a constant $C_{N, q, n}$, which depends only on $N, q$ and $n$, such that

$$
C_{N, q, n} \geqslant \frac{1}{|D|} \int_{D^{\#}} e^{N\left\langle\alpha_{q} u^{\#}(x)\right\}} m_{N, q, n}(|x|) d x,
$$

where $\alpha_{q}=\left(\omega_{n-1} \cdot n^{q-1}\right)^{1 / q}$, and if $R=\operatorname{diam}\left(D^{\#}\right), r=|x|$, we have

(a) $m_{N, 1, n}(r)=R^{n} e^{-N\left(r^{1-n}\right)} \cdot N^{\prime}\left(r^{1-n}\right) \cdot 1 / r^{2 n-1}$,

(b) $m_{N, n, n}(r)=R^{n} e^{-N\left\{\log \left(R^{n} / r^{n}\right)\right\}^{n /(n-1)}} \cdot N^{\prime}\left\{\log \left(\frac{R^{n}}{r^{n}}\right)\right\}^{n /(n-1)} \cdot\left\{\log \left(\frac{R^{n}}{r^{n}}\right)\right\}^{-1 / n}$,

(c) for $1<q<n, m_{n, q, n}(r)$ is bounded below by

$$
\begin{aligned}
R^{n} \exp [ & \left.-N\left\{\left(\frac{n(q-1)}{n-q}\right)^{(q-1) / q} \cdot r^{((q-n) / q)}\right\}\right] \\
& \cdot N^{\prime}\left\{\left(\frac{n(q-1)}{n-q}\right)^{(q-1) / q} \cdot\left[r^{(q-n) /(q-1)}-R^{(q-n) /(q-1)}\right]^{(q-1) / q}\right\} \\
& \cdot \frac{1}{\left(r^{(n+(n-q) / q)}\right)} .
\end{aligned}
$$


Proof. We prove (c) of the theorem noting that (a) and (b) are similar.

From Lemma $\mathrm{C}$ we have

$$
\int_{D^{\#}}\left|\nabla u^{\#}(x)\right|^{q} d x \leqslant \int|\nabla u(x)|^{q} d x \leqslant 1 .
$$

Define a variable $t$ by

$$
|r / R|^{n}=e^{-t}, \quad \text { where } R=\text { diameter of } D^{\#} .
$$

Let $f(t)=\alpha_{q} u^{\#}(r)$. We compute

$$
\left\{f^{\prime}(t)\right\}^{q}\left|\frac{d t}{d r}\right|^{q}=\left(\alpha_{q}\right)^{q}\left|\frac{d u^{\#}(r)}{d r}\right|^{q}
$$

or

$$
\left\{f^{\prime}(t)\right\}^{q}\left|\frac{n}{R} e^{t / n}\right|^{q}=\left(\alpha_{q}\right)^{q}\left|\nabla u^{\#}(r)\right|^{q} .
$$

We note that $f(0)=0$ from supp $u^{\#} \subset D^{\#}$, and $f^{\prime}(t) \geqslant 0$ for $t>0$. Thus,

$$
\begin{aligned}
(-) \int_{0}^{\infty}\left[f^{\prime}(t)\right]^{q}\left|\frac{n}{R} e^{t / n}\right|^{q} d\left\{R^{n} e^{-t}\right\} & =\left(\alpha_{q}\right)^{q} \cdot n \int_{0}^{R}\left|\nabla u^{\#}(r)\right|^{q} r^{n-1} d r, \\
& \leqslant\left(\alpha_{q}\right)^{q} \frac{n}{\omega_{n-1}} \cdot(1)=1 \cdot n^{q} .
\end{aligned}
$$

Define a weighted measure $\mu(t)$ by

$$
d \mu(t)=e^{-((n-q) / n) t} R^{n-q} d t,
$$

and let

$$
\phi_{\rho}(t)=e^{((n-q) / n) t} \cdot R^{q-n} \cdot \chi_{[0, \rho]}(t) .
$$

Then

(i) $\int_{0}^{\infty}\left[f^{\prime}(t)\right]^{q} d \mu(t) \leqslant 1$,

(ii) $\int_{0}^{\infty} f^{\prime}(t) \cdot \phi_{\rho}(t) d \mu(t)=f(\rho) \equiv F(\rho)$, and

(iii)

$$
\begin{aligned}
\psi(\rho) & =\left\|\phi_{\rho}\right\|_{q /(q-1), \mu(t)}=\left[\int_{0}^{\rho} \frac{e^{(1 /(q-1))((n-q) / n) t}}{R^{(n-q) /(q-1)}} d t\right]^{(q-1) / q} \\
& =R^{(q-n) / q}\left[\frac{n(q-1)}{n-q}\right]^{((q-1) / q)}\left[e^{(n-q) \rho / n(q-1)}-1\right]^{(q-1) / q} .
\end{aligned}
$$

We apply Theorem 3 with $1<q<n, \Phi(t)=e^{-t}, N$ a $C^{*}$-convex function, and (i), (ii), (iii) above to get

$$
C_{q} \int_{0}^{\infty} e^{-t} d t \geqslant \int_{0}^{\infty} e^{-N\langle\psi(\rho)\rangle+N\langle F(\rho)\}} d N\{\psi(\rho)\},
$$

where $C_{q}$ depends only on $N$ and $q$. We compute

$$
C_{q} \geqslant-\int_{0}^{\infty} e^{N\langle F(\rho)\rangle} \cdot\left[e^{-N\{\psi(\rho)\rangle} \cdot N^{\prime}\{\psi(\rho)\} \cdot e^{+\rho}\right] \psi^{\prime}(\rho) d e^{-\rho},
$$


and changing variables according to (1), this is

$$
C_{q} \geqslant \frac{1}{\omega_{n-1}} \frac{n}{R^{n}} \int_{S^{n-1}} \int_{0}^{R} e^{N\left\langle\alpha_{q} u^{\#}(r)\right\}} m_{N, q, n}(r) r^{n-1} d r d \sigma,
$$

where, in terms of the variable $\rho$,

$$
m_{N, q, n}(\rho)=e^{-N\{\psi(\rho)\}} \cdot N^{\prime}\{\psi(\rho)\} \cdot \psi^{\prime}(\rho) \cdot e^{\rho} .
$$

We compute, according to relation (1),

$$
\begin{aligned}
& e^{\rho}=(R / r)^{n} \\
& \psi^{\prime}(\rho)= R^{((q-n) / q)}\left(\frac{q-1}{q}\right)\left(\frac{n-q}{n(q-1)}\right)^{1 / q} \\
& \cdot[R / r]^{(n-q) /(q-1)}\left[(R / r)^{(n-q) /(q-1)}-1\right]^{-1 / q} \\
& \geqslant C_{n, q} r^{(q-n) / q}
\end{aligned}
$$

and

$$
\begin{aligned}
\psi(\rho) & =R^{((q-n) / q)}\left(\frac{n(q-1)}{n-q}\right)^{(q-1) / q} \cdot\left[(R / r)^{(n-q) /(q-1)}-1\right]^{(q-1) / q} \\
& \leqslant\left(\frac{n(q-1)}{n-q}\right)^{(q-1) / q} \cdot r^{(q-n) / q} .
\end{aligned}
$$

Thus

$$
\begin{aligned}
m_{N, q, n}(r) & \geqslant C_{q, n} R^{n} \exp \left[-N\left\{\left(\frac{n(q-1)}{n-q}\right)^{(q-1) / q} \cdot r^{(q-n) / q}\right\}\right] \\
\cdot & N^{\prime}\left\{\left(\frac{n(q-1)}{n-q}\right)^{(q-1) / q}\left[r^{(q-n) /(q-1)}-R^{(q-n) /(q-1)}\right]^{(q-1) / q}\right\} \cdot \frac{1}{r^{n+(n-q) / q}} .
\end{aligned}
$$

6. The theorems presented here are generalizations to those found in M. Jodeit [2]. Let $1<q<\infty, 1 / p+1 / q=1$, and $f$ be Lebesgue measurable on $(0, \infty)$ with $\int_{0}^{\infty}|f(x)|^{q} d x \leqslant 1$. Let $N$ be $C^{*}$-convex and $F(x)=\int_{0}^{x} f(t) d t$. Then, Theorem 3 implies that there exists a constant $C_{q, N}$ depending on $q$ and $N$ with

$$
\int_{0}^{\infty} e^{N(F(x))-N\left(x^{1 / p}\right)} d N\left(x^{1 / p}\right) \leqslant C_{q, N} .
$$

A substitution $x=\left[N^{-1}(\log (1 / u))\right]^{p}$ gives the following

Theorem 5. Let $N$ be $C^{*}$-convex and $g$ measurable on $I=(0,1)$ with

$$
1 \geqslant \int_{0}^{1} g^{q}(u) \frac{p\left[N^{-1}(\log (1 / u))\right]^{p-1}\left(N^{-1}\right)^{\prime}[(\log (1 / u))]}{u} d u .
$$

If, for $0<x<1$,

$$
T g(x)=\int_{x}^{1} g(u) \frac{p\left[N^{-1}(\log (1 / u))^{p-1}\left(N^{-1}\right)^{\prime}(\log (1 / u))\right]}{u} d u,
$$

then $\int_{0}^{1} e^{N(T g(x))} d x \leqslant C_{q, N}$. 
Since $g \rightarrow T g$ is a linear map from $L^{q}\left(I, d\left[N^{-1}(\log (1 / u))\right]^{p}\right)$ into $L_{A}(I, d x)$, where $L_{A}$ is the Orlicz space of norm $e^{N(x)}-1$, then the transpose of $T$ is a bounded linear map from $L_{\vec{A}}(I, d x)$ into $L^{p}\left(I, d\left[N^{-1}(\log (1 / u))\right]^{p}\right)$. We calculate that $L_{A}^{-}$is the Orlicz space of norm equivalent to $x\left[N^{-1}\left(\log ^{+}(x)\right)\right]$, and the transpose of $T$ is $S f(x)=\int_{0}^{x} f(t) d t$. Hence we have the following

Theorem 6. Let $N$ be $C^{*}$-convex, $1<p<\infty, \int_{0}^{1} f(t)\left[N^{-1}\left(\log ^{+} f(t)\right)\right] d t \leqslant 1$ and $S f(x)=\int_{0}^{x} f(t) d t$. Then there exists a constant $K_{p, N}$ which depends only on $N$ and $p$ such that

$$
\int_{0}^{1}(S f(u))^{p} \frac{p\left[N^{-1}(\log (1 / u))\right]^{p-1}\left(N^{-1}\right)^{\prime}[(\log 1 / u)]}{u} d u \leqslant K_{p, N} .
$$

\section{REFERENCES}

1. R. A. Hunt, On $L(p, q)$ spaces, Enseign. Math. 12 (1966), 249-276.

2. M. Jodeit, An equality for the indefinite integral of a function in Lq, Studia Math. 44 (19972). 545-554.

3. B. F. Jones, A note on Holder's inequality, Studia Math. 64 (1979), 272-278.

4. J. Moser, A sharp form of an inequality by N. Trudinger. Indiana Univ. Math. J. 20 (1971), 1077-1092.

5. G. D. Mostow, Quasi-conformal mappings in a n-space and the rigidity of hyperbolic space forms, Inst. Hautes Etudes Sci. Publ. Math. 34 (1968), 82-88.

6. C. J. Neugebauer, Some inequalities related to Hölder's inequality, Proc. Amer. Math. Soc. 82 (1981), $560-564$.

Department of Mathematics, Purdue University, West Lafayette, Indiana 47907

Current address: Department of Mathematical Sciences, Florida International University, Miami, Florida 33199 